may never be more than anecdotal. The allograft aortic root replacement option for a failed Ross procedure without reuse of the autograft gives the patient two allografts with potential for degeneration and need for subsequent technically demanding reoperations.

Our own experience with reoperation after the Ross procedure now exceeds 30 patients. Two thirds of these reoperations have related primarily to autograft failure; in retrospect, many of these patients could have benefited from the reversal described. Patients have also expressed psychologic relief. At this early stage, we are hopeful that the reversal procedure is a good, durable option for many patients with a failed Ross procedure.

\section{References}

1. Stelzer P, Elkins RC. Pulmonary autograft: an American experience. J Card Surg. 1987;2:429-33.

2. David TE, Omran A, Ivanov J, Armstrong S, de Sa MP, Sonnenberg B, et al. Dilation of the pulmonary autograft after the Ross procedure. J Thorac Cardiovasc Surg. 2000;119:210-20.

3. Kouchoukos NT, Masetti P, Nickerson NJ, Castner CF, Shannon WD, Davila-Roman VG. The Ross procedure: long-term clinical and echocardiographic follow-up. Ann Thorac Surg. 2004;78:773-81; discussion 773-81.

4. Leyh RG, Kofidis T, Fischer S, Kallenbach K, Harringer W, Haverich A. Aortic root reimplantation for successful repair of an insufficient pulmonary autograft valve after the Ross procedure. $J$ Thorac Cardiovasc Surg. 2002;124:1048-9.

\title{
Long abdominal aortic stenosis: A rare presentation of Takayasu arteritis treated with percutaneous stent implantation
}

\author{
Pasquale Perrone-Filardi, MD, Pierluigi Costanzo, BS, Paolo Cesarano, MD, Antonio Marzano, MD, \\ Giovanni Esposito, MD, Gabriele Iannelli, MD, and Massimo Chiariello, MD, Naples, Italy
}

$\mathrm{W}$ e describe the case of a patient with a long abdominal aortic stenosis. This condition can have different causes. On the basis of the American College of Rheumatology criteria and Sharma's diagnostic criteria, the cause in this patient was a rare presentation of Takayasu arteritis. Generally, this vasculitis affects the epiaortic arteries, causing diminished or absent pulses in the upper limbs; in this case, the arteritis affected the abdominal aorta, causing claudication of the lower limbs.

\section{Clinical Summary}

A 46-year-old woman was admitted to our department for claudication of the lower limbs. The claudication started 6 years before at the right limb and then progressed to the left limb, reaching a maximal walking distance of less than $100 \mathrm{~m}$ at the time of hospitalization. An echo-Doppler of the lower limbs demonstrated the absence of obstructive lesions. We performed an abdominal angio-computed tomographic scan with volumetric reconstruction that showed a long stenosis of the infrarenal aorta with a maximum diameter of less than $1.5 \mathrm{~cm}$ (Figure 1,

From the Department of Internal Medicine, Cardiovascular and Immunological Sciences, Federico II University, Naples, Italy.

Received for publication Jan 22, 2007; accepted for publication Jan 31, 2007.

Address for reprints: Pasquale Perrone Filardi, Via S. Pansini 5, Naples, 80131 Italy (E-mail: fpperron@unina.it).

J Thorac Cardiovasc Surg 2007;133:1647-8

$0022-5223 / \$ 32.00$

Copyright $\odot 2007$ by The American Association for Thoracic Surgery doi:10.1016/j.jtcvs.2007.01.063
$A, B)$. An abdominal echo-Doppler showed a long fibrotic plaque causing an obstruction of $40 \%$ of the lumen, with significant acceleration of speed without lesions at the renal and iliac arteries. Echo-Doppler of the carotid and subclavian arteries was normal. An angiographic study of the coronary and aortic tree showed multiple coronary stenosis that was hemodynamically not significant but atypical for the age and normal lipidemic profile of the patient $(50 \%$ stenosis of proximal left descending anterior artery, $40 \%$ stenosis of medium left descending anterior artery, and 50\% stenosis of medium circumflex artery). In the infrarenal aorta the angiographic study showed a hemodynamically significant long narrowing lesion (Figure 2, A). The assessment of inflammatory indexes revealed a high erythrocyte sedimentation rate. Finally, a percutaneous transluminal angioplasty with successful stent implantation was performed at the level of the infrarenal aorta (Figure 2,B) with immediate cessation of symptoms.

\section{Discussion}

Congenital coarctation of the thoracic aorta at the ligamentum arteriosum or aortic arch is well recognized. A much less common variety $(0.5 \%-2.0 \%)$ of aortic coarctation is located in the distal thoracic aorta, abdominal aorta, or both, and is usually called "middle aortic syndrome." 1 This definition includes different causes: congenital, Takayasu arteritis, neurofibromatosis, retroperitoneal fibrosis, and mucopolysaccharidosis. In our case, we excluded the last 3 diseases and considered a congenital coarctation or Takayasu arteritis. Some authors distinguish the abdominal localization of Takayasu arteritis from the congenital coarctation on the basis that the aortic stenosis in Takayasu arteritis affects a long tract of the infrarenal aorta without involvement of the renal arteries, whereas the aortic 

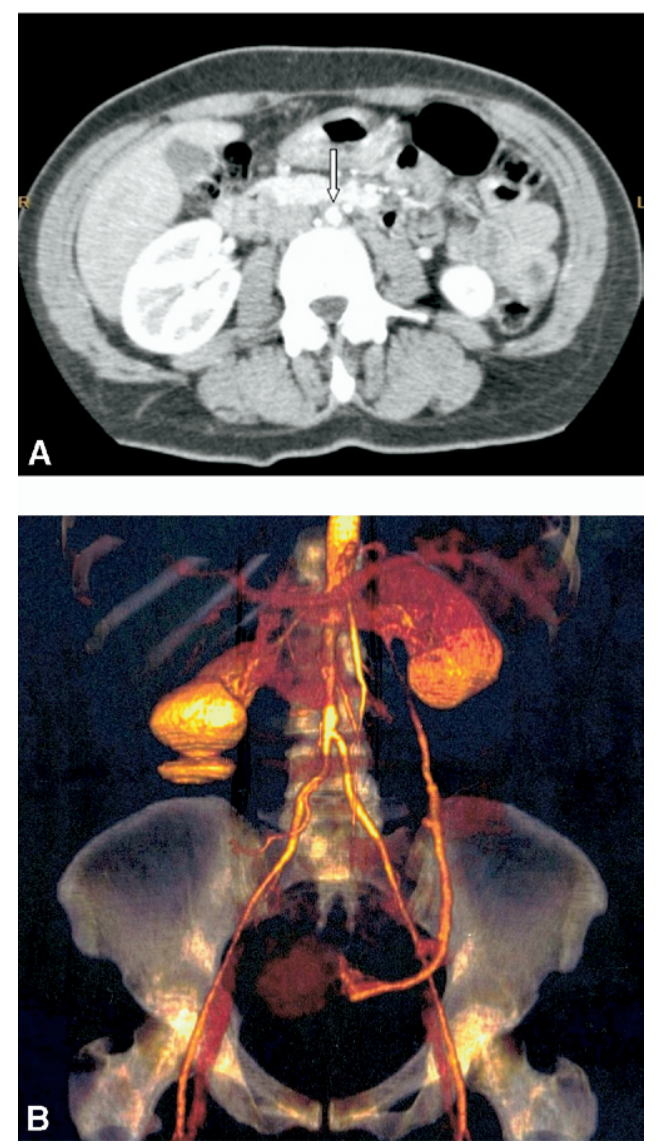

Figure 1. Abdominal angio-computed tomographic scan shows reduced lumen of the infrarenal aorta (arrow) with a maximum diameter of less than $1.5 \mathrm{~cm}$ (A). Volumetric reconstruction shows the long stenosis from the infrarenal aorta up to the iliac arteries (B).

stenosis in congenital coarctation affects the suprarenal aortic tract with involvement of the renal arteries. In our case, in addition to that, the angiographic result (long abdominal stenosis and coronary lesions) in a normolipidemic patient, with a high erythrocyte sedimentation rate, suggested Takayasu arteritis. According to the American College of Rheumatology criteria for this arteritis, 4 of the 6 criteria were met (age at disease onset $<40$ years, claudication of extremities, bruit abdominal aorta, and arteriogram abnormality; sensitivity of $90.5 \%$ and specificity of $97.8 \%$ ). According to Sharma's criteria, 1 major criterion (characteristic signs and symptoms of at least 1 month) and 3 minor criteria (high erythrocyte sedimentation rate, abdominal aorta lesion, and coronary artery lesion in absence of risk factors such as hyperlipidemia or diabetes mellitus) were met (sensitivity of $92.5 \%$ and specificity of $95 \%$ ). ${ }^{2}$
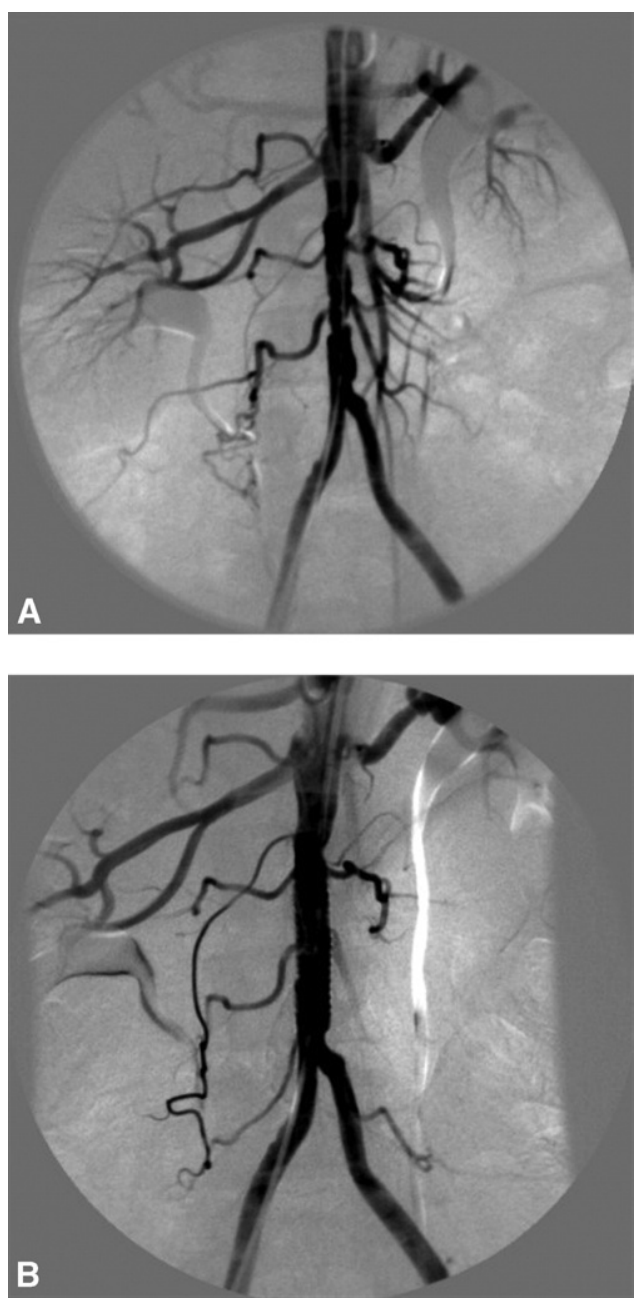

Figure 2. Aortographic scan shows the angiographic configuration of infrarenal aorta before (A) and after stent implantation (B).

Even if the treatment of the atypical congenital coarctation and the abdominal localization of Takayasu arteritis is the same (percutaneous transluminal angioplasty with stent implantation), it is important to distinguish between the two diseases. Unlike congenital coarctation, Takayasu arteritis is a chronic condition that needs to be monitored and, in case of relapse, treated with corticosteroid or immunosuppressive drugs.

\section{References}

1. Connolly JE, Wilson SE, Lawrence PL, Fujitani RM. Middle aortic syndrome: distal thoracic and abdominal coarctation, a disorder with multiple etiologies. J Am Coll Surg. 2002;194:774-81.

2. Sharma BK JS, Suri S, Numano. Diagnostic criteria for Takayasu arteritis. Int J Cardiol. 1996;54(Suppl):141-7. 